\title{
História da cirurgia da hipófise
}

\author{
Alexandre de Carvalho Galdino*, João Klescoski Junior**, José Alberto Landeiro*** \\ Serviço de Neurocirurgia do Hospital de Força Aérea do Galeão. Rio de Janeiro, Brasil.
}

\section{RESUMO}

O conhecimento da glândula hipófise e sua patologia impulsionaram o desenvolvimento da neurocirurgia. Sua localização única, entre a cavidade craniana e as nasais, e a combinação de sintomatologia neuro, endocrino e oftalmológica causada por tumores da região, motivaram o emprego de diversas técnicas cirúrgicas. Apresentamos aqui os principais marcos históricos relacionados com o desenvolvimento da cirurgia da região selar e para-selar.

\section{PALAVRAS-CHAVE}

História da neurocirurgia. Cirurgia da hipófise. Cirurgia transesfenoidal.

\section{ABSTRACT \\ Historical aspects of the pituitary gland surgery paraselar neurosurgical evolution. \\ KEY-WORDS \\ History of neurosurgery. Pituitary surgery. Transsphenoidal surgery.}

The knowledge of pituitary gland and its associated pathologies led to the development of early neurosurgery. The pituitary's unique position - between the cranial and the nasal cavities - and the combination of neurological, ophtalmological, and endocrinological symptoms produced by the pituitary tumors led to the development of several surgical methods. The authors make a historical overview on selar and

\section{Conhecimento da glândula}

Os primeiros relatos históricos sobre a hipófise remontam à Grécia Antiga, embora a função da glândula tenha ficado desconhecida até o final do século XIX ${ }^{14,44}$. Flesch ${ }^{16}$ sugeriu, em 1885, não ser a hipófise um remanescente filogenético rudimentar como até então se acreditava. Idéia essa reforçada por Rogowitsch $^{52}$, de Kiev, em 1889, quando observou o aumento substancial da glândula após tireoidectomia total, acreditando ser a hipófise uma glândula sem ducto que secretaria diretamente na corrente sangüínea. Tal observação incitou modelos experimentais, sendo a hipofisectomia em animais finalmente descrita por Nicholas Paulesco (1869-1931) ${ }^{51}$, técnica posteriormente revista e aprimorada por Cushing e Cowe ${ }^{8}$ com análise microscópica e estudos de necropsia.
Paulesco $^{51}$ observou que a maioria dos cães morria em 24 horas após hipofisectomia transcraniana total, e que a ressecção parcial não alterava a sobrevida dos animais. Notou ainda que a hipofisectomia total acarretava uma série peculiar de características denominada caquexia "hipofisepriva".

Outras manifestações observadas foram adiposidade, hipoplasia secundária de órgãos sexuais, infantilismo sexual persistente, poliúria, glicosúria, alterações cutâneas (edema e hipotricose), hipotermia e letargia. Tais achados também foram observados após a separação da hipófise da base do cérebro e após adeno-hipofisectomia seletiva. Cushing e col. ${ }^{8}$ tiveram experiência similar e notaram ainda o aumento da sobrevida dos animais hipofisectomizados após injeções de extratos glandulares e emulsões de hipófises canina, ovina e bovina, e por auto e homotransplante de tecido

\footnotetext{
* Médico residente do Serviço de Neurocirurgia do Hospital de Força Aérea do Galeão.

** Médico do Serviço de Neurocirurgia do Hospital de Força Aérea do Galeão.

*** Chefe do Serviço de Neurocirurgia do Hospital de Força Aérea do Galeão.
} 
hipofisário sob o músculo reto abdominal. Iniciou ainda a administração oral e hipodérmica de extrato bovino a pacientes com dispituitarismo (hipopituitarismo) secundário a tumor ou destruição cirúrgica da glândula.

Em contrapartida, a promoção de hiperpituitarismo experimental foi realizada por Evans e Long ${ }^{15}$, em 1922, e tal condição recebia a denominação geral de acromegalia, termo adotado por Pierre Marie (1853-1940) a partir da observação semiológica, que encontrou embasamento patológico após descrição de achados em necropsia por Marinesco (1863-1938) em um paciente acromegálico ${ }^{44}$.

A existência de um tumor hipofisário corretamente nomeado adenoma já havia sido observada em 1884, por Frietzsche e Klebs, constante achado no gigantismo. Em 1890, Souza Leite, discípulo de Pierre Marie, havia coletado 38 casos de adenomas. Em Nova York, Woods Hutchinson notou, em 19 pacientes com gigantismo e acromegalia, o alargamento uniforme do lobo anterior da glândula.

Em 1910, a amenorréia-galactorréia foi observada por Oskar Hirsch (1877-1965) $)^{31}$ em uma mulher de 35 anos, com constatação cirúrgica de um cisto local e observação de um adenoma em necropsia. No ano seguinte, Hirsch diferenciou as três entidades clínicas mais importantes até então conhecidas: acromegalia, distrofia adiposogenital (descrita por Alfred Frohlich) e uma terceira condição caracterizada por alterações visuais. Em 1933, Harvey Cushing (1869-1939) ${ }^{10}$ descreveu o caso de uma paciente com amenorréiagalactorréia persistente após o parto, suspeitando, então, de hipersecreção de hormônio lactogênico, a prolactina (isolada no ano seguinte por Riddle). A síndrome de amenorréia-galactorréia e baixos níveis de FSH (síndrome causada por hiperprolactinemia) foram posteriormente mais bem caracterizados por Argonz e Castillo $^{2}$, em 1953, e Forbes e col. ${ }^{17}$, em 1954.

Em 1912, Cushing ${ }^{11}$ introduziu o termo síndrome poliglandular, aludindo ao caso de uma paciente de 23 anos de idade com obesidade, hipertricose e amenorréia. Coletando 15 outros casos com sintomas semelhantes na literatura e casos próprios, concluiu que alguns definitivamente eram determinados por tumor adrenal com melhora após ressecção, porém, que uma síndrome similar poderia acompanhar adenoma basófilo da hipófise associada à hiperplasia das glândulas adrenais, o que é conhecida hoje por doença de Cushing.

A possibilidade de determinar o nível sérico dos hormônios abriu novos horizontes para a detecção precoce de adenomas hipofisários e seu acompanhamento. $\mathrm{O}$ estudo por rádio-imunoensaio do hormônio do crescimento foi introduzido por Utiger ${ }^{56} \mathrm{em} \mathrm{1962,}$ do ACTH por Yalow ${ }^{58}$ em 1964 e da prolactina em 1971 por Hwang ${ }^{34}$.

\section{Acessos transcranianos}

Três foram os objetivos iniciais nas abordagens transcranianas: descompressão subtemporal para diminuição da pressão intracraniana e alívio de cefaléia; descompressão supra-selar tumoral para melhora da visão; tratamento do hiperpituitarismo (acromegalia, até então a única entidade associada à hiperfunção da glândula) $)^{1,44}$.

A primeira cirurgia à região selar foi realizada, em

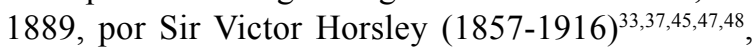
visando à retirada de massa pré-quiasmática. Após craniotomia frontal, retração do lobo frontal e visualização do processo expansivo, julgou tratar-se de adeno-sarcoma cístico de pituitária, considerando a lesão irressecável. A paciente morreu anos depois e, à necropsia foi verificado amolecimento do lobo frontal, associada possivelmente à ligadura de veias frontais que drenavam para o seio sagital superior, fazendo com que Horsley abandonasse tal técnica, passando a adotar o acesso temporal. Entre 1904 e 1906, Horsley operou dez tumores hipofisários usando tanto o acesso subfrontal quanto o temporal, com mortalidade de $20 \%{ }^{48}$.

Bem documentada foi a paciente de Frank Paul (1851-1941) ${ }^{50}$, em 1893, na cidade de Liverpool. Tratava-se de uma médica de 30 anos de idade do hospital onde trabalhava, com história de três anos de evolução de acromegalia, cefaléia progressiva e perda de visão, que foi submetida à craniotomia temporal descompressiva, por sugestão de Horsley ${ }^{60}$.

Em Nova York, no ano de 1903, Otto George Theobald Kiliani ${ }^{38}$ operou, em caráter de emergência, um paciente com quadro de apoplexia hipofisária, com hemorragia intratumoral associada à hematoma subdural agudo pelo acesso bifrontal (..." the patient was so comatous that it was deemed possible to operate without narcose"...).

Em janeiro de 1900, Fedor Victor Krause ${ }^{41}$, em Berlim, realizou com sucesso a retirada de um projétil de arma de fogo de um jovem suicida de 20 anos, utilizando o acesso transfrontal, sugerindo, a partir de então, tal via para lesões hipofisárias. Em 1905, realizou pela primeira vez o acesso transfrontal para tumor hipofisário, optando pela via extradural, com abertura de dura-máter apenas com $5 \mathrm{~cm}$ de profundidade, para obter acesso ao tumor e ao quiasma ${ }^{42}$. Posteriormente, adotou o acesso frontolateral intradural por onde seria possível retirar o tumor "com o dedo indicador e uma cureta grande" 43 . Finalmente, viria a adotar o acesso transnasal superior descrito por Schloffer, deixando o acesso transcraniano para tumores com grandes extensões inabordáveis por via transesfenoidal.

Braun $^{5}$, em 1907, após estudos em cadáveres, estava convencido que a partir do acesso transtemporal através 
do seio cavernoso, após seccionar o ramo maxilar do nervo trigêmeo e ligar a artéria carótida interna cervical, poder-se-ia acessar a hipófise.

Louis Linn McArthur (1858-1934) ${ }^{49}$, em Chicago, no ano de 1908, descreveu o acesso frontorbitário extradural, com abertura da dura-máter a $0,5 \mathrm{~cm}$ do sulco do quiasma. Tal acesso também foi realizado por Charles Frazier (1870-1930) ${ }^{18}$, na Filadélfia, adotando posteriormente abordagem intradural. Frazier apresentava experiência na abordagem transnasal de lesões da hipófise, destinando o acesso transesfenoidal para lesões unicamente intra-selares, estando convencido que, na presença de extensão supra-selar e perda visual, o melhor acesso seria sempre o transcraniano.

Em 1910, após estudos anatômicos e hipofisectomias experimentais, Silbermark ${ }^{54}$ sugeriu o acesso através da fissura silviana para lesões da hipófise. Em 1914, George Heuer (1882-1950) ${ }^{29}$ de Baltimore, Maryland, descreveu o acesso transcraniano intradural ao quiasma que diferia dos acessos de Krause e McArthur. Em 1917, em virtude de uma viagem urgente à França, Heuer legou a Walter Dandy a apresentação de sua casuística de 20 cirurgias utilizando tal acesso, onde o tumor poderia ser atingido ao longo da asa do esfenóide, através da fissura silviana ${ }^{13}$. A mesma técnica foi utilizada por Alfred Adson (1887-1951) na Clínica Mayo ${ }^{1}$.

\section{Acesso transesfenoidal}

\section{Transnasal superior}

Davide Giordano (1864-1954) ${ }^{19}$, em Veneza, realizou estudos em cadáveres visando ao acesso inferior (transesfenoidal) à sela túrcica, em 1897. Acreditava que os acessos transtemporal e supraorbitário não eram viáveis, pela alta morbidade que acarretavam, particularmente a retração do lobo frontal e possível injúria do quiasma.

Em 16 de março de 1907, em Innsbruck, na Áustria, Hermann Schloffer ${ }^{37,44,45,47,48,53}$ realizou a primeira cirurgia transesfenoidal à hipófise. Motivado pelo fracasso surgido da inviabilidade de tratar dois pacientes prévios que sucumbiram à mesma doença, Schloffer realizou antes do procedimento estudos topográficos em cinco cadáveres com adenomas hipofisários obtidos de Hans Chiari (1851-1916) em Praga e um caso de Anton Weichselbau (1845-1920) em Viena.

Tratava-se de um homem de 30 anos de idade com quadro de hipopituitarismo, cefaléia, vômitos, distúrbios visuais, hipotricose, impotência e perda da libido com cerca de dois anos de evolução. Ao exame, apresentava hemianopsia bitemporal, paresia facial à esquerda e alargamento selar na radiografia do crânio. Sob anestesia local com cocaína, Schloffer realizou a cirurgia em três estágios, representando aparentemente uma modificação dos acessos contemporâneos para tratar sinusite esfenoidal. Após rinotomia com reflexão do nariz e septo ósseo para a direita, foi realizada ressecção da parede medial da órbita, septo nasal, cornetos médios com parte da parede do seio maxilar e seios etmoidais, com exposição e abertura da face anterior do seio esfenóide. Dificuldade foi encontrada na identificação intraoperatória da sela túrcica, problema este contornado pela mensuração, na radiografia, da distância da glabela à face anterior da sela alargada. Realizou osteotomia do assoalho selar adelgaçado pela lesão e subseqüente escavação tumoral, com constatação de inadvertida fístula liquórica. A cavidade tumoral abordada foi fechada com tampão embebido em bálsamo proveniente do Peru. O paciente apresentou melhora do quadro de cefaléia, porém, persistência da hemianopsia. Evoluiu com sinais de hipertensão intracraniana e, em 22 de maio (dois meses após a cirurgia) ocorreu o óbito. A necropsia evidenciou hidrocefalia com obstrução do forame interventricular por uma grande extensão tumoral supraselar, muito maior do que supunha Schloffer.

Ainda em 1907, poucos meses após, o mesmo acesso transnasal superior foi realizado por Anton von Eiselsberg (1820-1939), Stumme e Julius von Hochenehh (1859-1940) de Viena, e por Borchardt em Berlim ${ }^{44,48}$. Vale lembrar que von Eiselsberg, apenas três meses depois, realizou a cirurgia em um jovem de 20 anos, caso original da distrofia adiposogenital de Frolich, com modificações da técnica de Schloffer, ocorrendo desnecessária exposição do seio frontal e ressecção de todos os seis cornetos nasais. O paciente, no entanto, apresentou melhora expressiva do quadro de cefaléia e recuperação do déficit visual, morrendo 15 anos após a cirurgia inicial.

Emil Teodor Kocher ${ }^{39,46}$ adotou uma medida que viria a ser um grande avanço na cirurgia transnasal, sugerindo a ressecção submucosa do septo nasal, permanecendo assim fora da cavidade nasal.

\section{Transnasal inferior}

Oskar Hirsch (1877-1965) ${ }^{31}$, um jovem rinologista de Viena, afirmou em um congresso local, em 1909, que a grande ressecção óssea facial promovida pelos acessos de Schloffer, von Eiselsberg e Hochenegg eram desnecessárias, e que eles, rinologistas, estavam habituados a abrir o seio esfenoidal por um acesso exclusivamente nasal, como Markus Hajek (18611941) havia demonstrado em 1904. Sua sugestão foi 
recebida inicialmente com ceticismo, porém Hirsch, no ano seguinte ${ }^{32}$, pode demonstrar sua técnica em uma paciente encaminhada pelo influente professor Julius Wagner von Jaureg, pessoa de caráter dominante que permitiu a Hirsch realizar a cirurgia em diversas etapas. Tratava-se de uma mulher de 35 anos de idade, com quadro de amenorréia-galactorréia há cerca de quatro anos, associada à cefaléia, amaurose à esquerda, visão residual em quadrante superior direito e crises convulsivas. O procedimento, sob anestesia local, foi iniciado em 26 de janeiro de 1910 com ressecção do corneto inferior esquerdo. $\mathrm{O}$ segundo passo foi ressecção das células etmoidais à esquerda, em $1^{\circ}$ de fevereiro, com subseqüente abertura da parede anterior do seio esfenoidal em 10 de fevereiro. Neste ponto, a paciente apresentou breve melhora do quadro visual. Osteotomia do assoalho da sela foi a etapa seguinte, em 26 de fevereiro, abertura da dura-máter sem remoção do tumor, em 8 de março, realizando apenas retirada de material para biópsia. Foram documentadas melhora da visão e campo visual. A análise histológica do que se julgava ser a parede cística do tumor mostrou tratarse de dura-máter e uma camada de hipófise normal. A paciente morreria 15 meses após a cirurgia em virtude de crises convulsivas e pneumonia. A necropsia revelou um adenoma pituitário de $3 \mathrm{~cm}$ x 2,5 $\mathrm{cm}$ x $1,5 \mathrm{~cm}$.

Em maio do mesmo ano, insatisfeito com os grandes intervalos do caso anterior, Hirsch optou por procedimento em tempo único, influenciado pela idéia de Kocher sobre cirurgia extracavitária, adotando a ressecção submucosa do septo de acordo com a recém descrita técnica elaborada por Gustav Killian (18601921). Tratava-se de uma mulher de 46 anos de idade com cefaléia, perda visual e galactorréia. Usando um espéculo nasal para manter o campo aberto, Hirsch abriu o seio esfenoidal, ressecou o septo esfenoidal, perfurou o assoalho selar e, ao incisar a dura-máter obteve drenagem espontânea de um tumor de consistência amolecida. Assim, ele criou a técnica de esfenoidotomia anterior direta que é usada até hoje.

Albert Edward Halstead (1869-1926) ${ }^{24}$, auxiliado por Allen Kanavel (1874-1938), realizou sua primeira cirurgia transesfenoidal em julho de 1909, a partir de uma incisão sublabial.

Harvey Cushing realizou sua primeira cirurgia transesfenoidal para adenoma pituitário em março de $1909^{9}$, usando a técnica de Giordano-Schloffer em seus dois primeiros pacientes, adotando a técnica de Halstead-Kanavel em seus cinco pacientes seguintes. A primeira cirurgia por ele realizada com a técnica, que levaria seu nome, ocorreu em junho de 1910, onde realizou incisão sublabial de Halstead e procedeu a ressecção submucosa do vômer. A paciente morreu no dia seguinte com quadro de hipertensão intracraniana causada por hidrocefalia aguda.

\section{Miscelânea transesfenoidal}

O acesso transpalatal, descrito por Fritz Koenig (1866-1952) $)^{40}$ nunca obteve muita popularidade. Foi realizado inicialmente por Ballance em 1908, tendo ocorrido severa hemorragia ao incisar a epifaringe e óbito do paciente cinco horas após o procedimento. Outras cirurgias, utilizando esta via, foram realizadas por Herman Preysing (1866-1926) em Colônia, Alemanha.

Idealizado por Marschik, assistente de Chiari, em 1910, o acesso extra-axial transetmoidal superior visava diminuir a profundidade de exposição da sela túrcica, a partir de uma incisão semicircular ao redor do ângulo medial do olho. Foi realizado pela primeira vez por Ottokar Chiari, em junho de $1911^{7}$.

Fein estudou o método transmaxilar (transantral) visando à maior exposição da glândula do que poderia ser obtida com os acessos de Schloffer e Hirsch. Usada inicialmente por Alfred Denker (1863-1941), foi simplificada em 1928 por Lautenschlager e revista em 1965 por Svien e Litzow ${ }^{55}$.

Apesar da grande popularidade da cirurgia da hipófise no início do século, ainda persistia grande a taxa de mortalidade cirúrgica. Uma dificuldade particular era acessar a extensão supra-selar do tumor e vários desastres sucederam explorações cirúrgicas, principalmente pela via transesfenoidal. Por esta razão, Bakulew ${ }^{4}$ sugeriu o uso pré-operatório da pneumoencefalografia, descrita por Walter Dandy em 1919, para confirmação do tumor e determinação de sua extensão supra-selar.

A hemostasia era outra dificuldade; grande avanço foi obtido com o advento da eletrocoagulação (unidade Bovie) introduzida por Cushing em $1927^{12}$. O uso de tampões era então prática comum, sendo removidos após períodos superiores a três semanas.

Tais dificuldades e pelo fato de haver melhor recuperação do déficit visual após cirurgias transcranianas ( $42 \%$ após transcraniana e $37 \%$ após transesfenoidais), levou Cushing e grande número de neurocirurgiões a abandonarem o acesso inferior à sela.

A série de Cushing totalizou 338 pacientes, 107 operados por acesso transcraniano e 231 por acesso transnasal, a despeito de sua preferência final pelo acesso transcraniano ${ }^{28}$. Sua taxa de mortalidade foi de $6,2 \%$. Outros neurocirurgiões tinham taxas igualmente altas: a de Grant ${ }^{20}$, em 1939, foi de $11,3 \%$ em 44 transesfenoidais e 9,3\% em transcranianas; em 1940, Jefferson $^{35}$ reportou $2 \%$ em 98 microadenomas e $33 \%$ em 12 macroadenomas; em 1950, Olivercrona ${ }^{3}$ reportou $8,9 \%$ em 303 cirurgias; Krayenbuhl27, em 1959, 
8,4\% em 106 cirurgias. Em virtude dessa alta taxa de mortalidade, neurocirurgiões tornaram-se altamente relutantes em operar adenomas pituitários, a despeito da redução de mortalidade cirúrgica em virtude da melhoria das técnicas anestésicas, antibióticos e o uso de cortisona. Deve-se lembrar que a taxa de mortalidade era ainda mais elevada quando em comparação com o tratamento radioterápico, o que levou à disseminação desta opção terapêutica.

\section{Ressurgimento do acesso transesfenoidal}

Rinologistas continuaram a utilizar o acesso transesfenoidal, ao contrário da grande maioria dos neurocirurgiões que, influenciados pela decisão de Cushing, abandonaram a técnica. Oscar Hirsch emigrou de Viena para os EUA em $1938^{60}$. Em 1957, publicou os resultados de uma série de 413 pacientes operados por via transesfenoidal ${ }^{30}$. Ele havia diminuído sua já então considerada baixa taxa de mortalidade de 4,8\% (19191929) para 1,4\% (1945-1956) com a combinação de cirurgia transesfenoidal e radioterapia local.

No entanto, a técnica transesfenoidal de Cushing foi mantida viva por seu discípulo Norman Dott (18971973) em Edimburgo (Figura 1), que aprimorou a visualização do sítio operatório ao instalar duas pequenas lâmpadas no espéculo nasal para hipófise idealizado por Cushing. Dott operou 120 adenomas hipofisários pelo acesso transesfenoidal sem mortalidade e, em

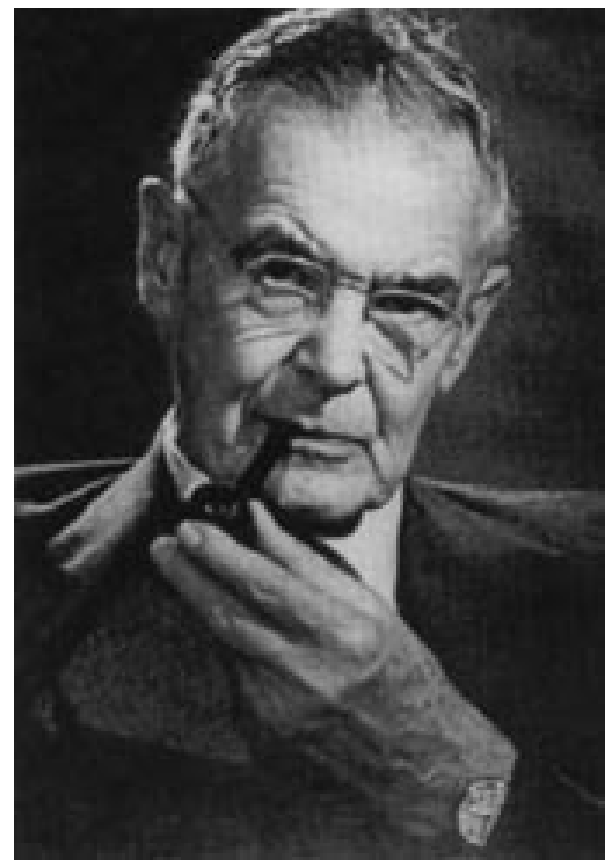

Figura 1 - Norman Dott, discípulo de Cushing. Manteve viva a chama da cirurgia transesfenoidal na $2^{a}$ Guerra Mundial. virtude da radioterapia, sem recorrência. Gerard Guiot (1912-1996) ${ }^{22}$ acreditava que Dott nunca publicou seus casos em deferência póstuma ao seu mestre, que autoritariamente havia condenado tal método. Dott introduziu Guiot à técnica de Cushing em 1956, durante uma jornada em Edimburgo. Guiot teve a oportunidade de observar Dott operando dois adenomas hipofisários por via transesfenoidal.

O novo interesse pela cirurgia hipofisária transesfenoidal, tal qual conhecemos após o final da década de 1960, originou-se em Paris no serviço de Guiot (Figura 2) no Hospital Foch, com propagação adicional da técnica por seu discípulo Jules Hardy em Montreal ${ }^{25,26}$. Guiot começou a operar de frente para o paciente, que

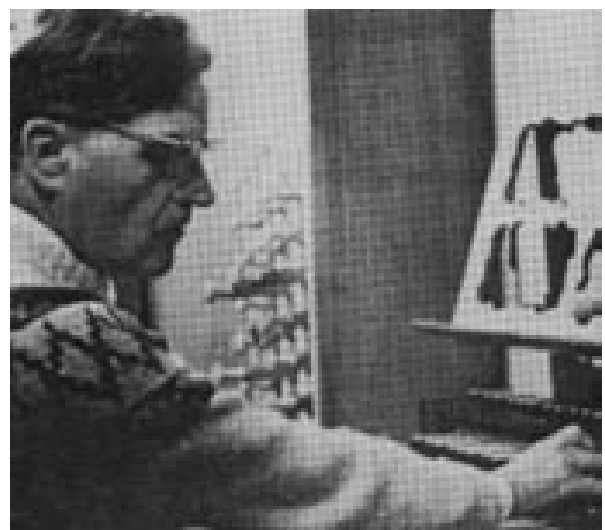

Figura 2 - Gerard Guiot, influenciado por Dott, introduziu o raio-X pré-operatório no acesso à sela.

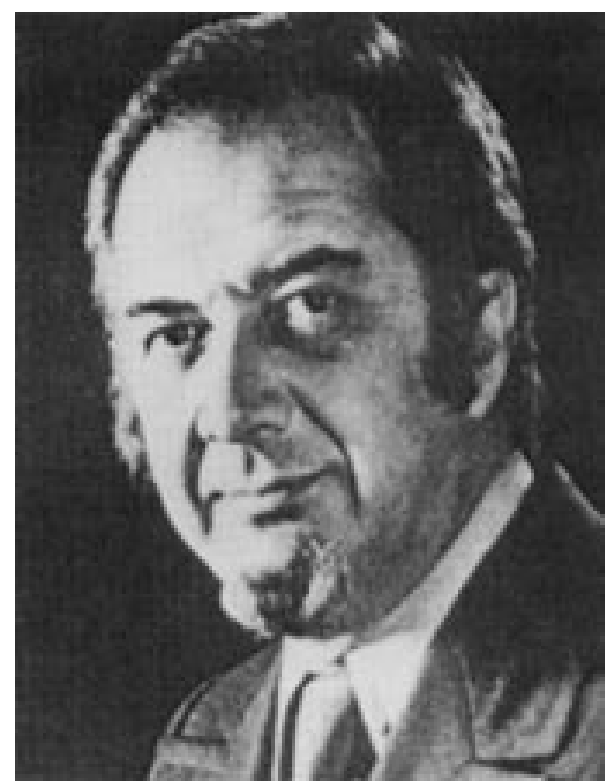

Figura 3 - Jules Hardy, de Montreal, introduziu o microscópio cirúrgico na cirurgia transesfenoidal. 
se encontrava na posição semi-sentada, ao contrário de Dott que operava sentado com a cabeça do paciente posicionada entre suas pernas. Guiot introduziu a radiografia intra-operatória e posteriormente a radioscopia (juntamente com Hardy). Em 1967, Hardy (Figura 3) introduziu o microscópio cirúrgico. Guiot preferia operar com lupa e luz frontal, embora utilizasse o microscópio cirúrgico com fins didáticos.

Estas novas técnicas determinaram um rápido crescimento no número de cirurgias transesfenoidais. Guiot realizou sua primeira cirurgia transesfenoidal em 1957, tendo operado antes disso 34 pacientes por via transcraniana. Em 1967, a percentagem de procedimentos transesfenoidais em seu serviço já atingia $72 \%^{21,23}$.

Outros refinamentos, incluindo a introdução do endoscópio, uso de guia estereotáxica e ressecções agressivas de base de crânio, proveram corredores cirúrgicos a tumores previamente considerados inacessíveis.

Os princípios da cirurgia da base do crânio foram extrapolados para cirurgia transesfenoidal por Martim Weiss $^{57}$, da Universidade do Sul da Califórnia, que publicou sua experiência com o acesso expandido em 1987, tendo sido revolucionário no tratamento de lesões como craniofaringeoma, meningeoma do tubérculo da sela e cisto da bolsa de Rathke.

O uso de endoscópio na cirurgia hipofisária foi pela primeira vez descrita por Bushe e Halves ${ }^{6}$ em 1978, porém, adquirindo popularidade apenas em meados dos anos 1990, quando virtualmente substituiu as técnicas convencionais no tratamento de doenças inflamatórias paranasais pelos otorrinolaringologistas, estimulando os neurocirurgiões a reexplorarem sua potencial aplicação na cirurgia transesfenoidal para tumores hipofisários. Yaniv e Rappaport ${ }^{59}$ descreveram um acesso combinado no qual o endoscópio foi usado no acesso inicial ao seio esfenoidal, com subseqüente conversão à ressecção microcirúrgica transesfenoidal convencional. Em 1997, Jho e Carrau ${ }^{36}$ publicaram resultados encorajadores de uma série de 50 pacientes portadores de tumores hipofisários submetidos à cirurgia transesfenoidal endonasal endoscópica.

Em suma, em uma época em que as pesquisas clínicas permitem tratar uma série de tumores hipofisários, a cirurgia ainda exerce um papel preponderante e, muitas das técnicas hoje utilizadas foram propostas há mais de século.

\section{Referências}

1. ADSON AW: Hypophysial tumors through the intradural approach. JAMA 71:721-6, 1918.

2. ARGONZ J, DEL CASTILLO EB: A syndrome characterized by estrogenic insufficiency, galactorrhea and decreased urinary gonadotropin. J Clin Endocrinol 13:79-87, 1953.
3. BAKAY L: The results of 300 pituitary adenomas operations (Prof. Hebert Olivercronas series). J Neurosurg 7:240-55, 1950.

4. BAKULEW AN: Zur diagnose und operativen Behandlung von Hypophysistumoren. Langenbecks Arch Klin Chir 139:679-98, 1926.

5. BRAUN H: Zur Freilegung der zentralen Teile der mittleren Schadelgrube (Ganglion Gasseri und Sinus cavernosus und der Hypophyse). Dtsch Z Chirurgie 87:130-59, 1907.

6. BUSHE KA, HALVES E: Modifizierte Technik bei transnasaler Operation der Hypophysengeschwulste. Acta Neurochir 41:163-75, 1978.

7. CHIARI O: Ueber eine Modifikation der Schlofferschen Operation von Tumoren der Hypophyse. Wien Klin Wochenschr 25:5-6, 1912.

8. COWE SJ, CUSHING H, HOMANS J: Experimental hypophysectomy. Johns Hopkins Hosp Bull 21:127-69, 1910.

9. CUSHING H: Partial hypophysectomy for acromegaly. With remarks on the function of the hypophysis. Ann Surg 50:1002-17, 1909.

10. CUSHING H: Dyspituitarism: twenty years later. With special consideration of pituitary adenomas. Arch Intern Med 51:487-557, 1933.

11. CUSHING H: The pituitary body and its disorders. Clinical states produced by disorders of the hypophysis cerebri. Pliladelphia, JB Lippincott, 1912; pp 341.

12. CUSHING H: Meningiomas arising from the olfactory groove and their removal by the aid of electro-surgery. Lancet 1:1329-39, 1927.

13. DANDY WE: A new hypophysis operation. John Hopkins Hosp Bull 29:154-5, 1918.

14. DAVEY LM: Early historical aspects of the pituitary gland. In Goodrich J, Lee KJ (eds): The Pituitary. Amsterdam, Elsevier Science, 1987, pp 1-17.

15. EVANS HM, LONG JA: Characteristic effects upon growth, oestrus, and ovulation induced by the intraperitoneal administration of fresh anterior hypophyseal substance. Proc Natl Acad Sci USA 8:38-9, 1922.

16. FLESCH M: Tageblatt 58 . Versammlung deutsche Naturforscher und Aertze, Strassburg, pp 411-412. Apud Romeis B: Hypophyse. In Mollendorff W (ed): Handbuch der Mikroskopischen Anatomie des Menschen. Berlim, J Springer, 1940, pp 5, 565 .

17. FORBES AP, HENNEMAN PH, GRISWOLD GC: Syndrome characterized by galactorrhea, amenorrhea and low urinary FSH: comparison with acromegaly and normal lactation. J Clin Endocrinol Metab 14:265-71, 1954.

18. FRAZIER CH: Lesions of the hypophysis from the viewpoint of the surgeon. Surg Gynecol Obstet 17:724-36, 1913.

19. GIORDANO D: Compendio di Chirurgia Operatoria. Torino, Unione Tipografico-Editrice Torinese, 1897, pp 100-104.

20. GUIOT FC: The surgical treatment of pituitary adenomas. JAMA 113:1279-82, 1939.

21. GUIOT G: Considerations on the surgical treatment of pituitary adenomas. In: Fahlbusch R, von Werder K (eds): Treatment of Pituitary Adenomas. Stuttgart, G Thieme, 1978, pp 202- 18.

22. GUIOT G, ARFEL G, BRION S: Adenomes Hypophysaires. Paris, Masson, 1958, pp 276.

23. GUIOT J, BOUCHE J, OPROIU A: Les indications de l'abord trans-sphenoidal des adenomas hypophysaires. Experience de 165 interventions. Presse Med 75:1563-8, 1967.

24. HALSTEAD AE: Remarks on operative treatment of tumors of hypophysis: with the report of two cases operated on by an oro-nasal method. Trans Am Surg Assoc 28:73-93, 1910.

25. HARDY J: Transphenoidal microsurgery of the normal and pathological pituitary. Clin Neurosurg 16:185-216, 1969.

26. HARDY J: Transsphenoidal hypophysectomy. J Neurosurg 34:582-94, 1971. 
27. HEIMBACH SB: Follow-up studies on 105 cases of verified chromophobe and acidophile pituitary adenomata after treatment by transfrontal operation and x-ray irradiation. Acta Neurochir 7:101-55, 1959.

28. HENDERSON WR: The pituitary adenomata. A followup study of the surgical results in 338 cases (Dr. Harvey Cushings series). Br J Surg 26:811-921, 1939

29. HEUER GJ: Surgical experiences with an intracranial approach to chiasmal lesions. Arch Surg 1:368-81, 1920.

30. HIRSCH O: Hypophysentumoren - ein Grenzgebiet. Acta Neurochir (Wien) 5:1-10, 1957

31. HIRSCH O: (Meeting of the k.k. society of physicians in Vienna, April 8, 1910). Wien Klin Wochenschr 23:563-5, 1910.

32. HIRSCH O: Endonasal nethod of removal of hypophyseal tumors. With report of two successful cases. JAMA 55:772-4, 1910.

33. HORSLEY V: On the technique of operations on the central nervous system. Br Med J 2:411-23, 1906.

34. HWANG P, GUYDAH, FRIESEN H: A radioimmunoassay for human prolactin. Proc Natl Acad Sci USA 68:1902-6, 1971.

35. JEFFERSON G: Extrasellar extensions of pituitary adenomas. Proc R Soc Med 33:433-58, 1940.

36. JHO HD, CARRAU RL: Endoscopic endonasal transsphenoidal surgery: experience with 50 patients. J Neurosurg 87:44-51, 1997

37. KANTER AS, DUMONT AS, ASTHAGIRI AR, OSKOUIAN RJ, JANE Jr JA, LAWS ER: The transsphenoidal approach - A historical perspective. Neurosurg Focus 18:E6, 2005

38. KILIANI OGT: Some remarks on tumors of the chiasm, with a proposal how to reach the same by operation. Ann Surg 40:35-43, 1904

39. KOCHER T: Ein Fall von Hypophysistumor mit operativer Heilung. Dtsch Z Chirurgie 100:13-37, 1909

40. KOENIG F: Zur totalestirpation der hypophysis cerebri. Berl Klin Wochenschr 37:1040, 1909

41. KRAUSE F: Schussverletzungen des Scadels. In von Leyden E, Klemperer F (eds): Die Deutsche Klinik am Eingange des Zwanzigsten Jahrhunderts, in Akademischen Vorlesungen. Berlim, Urban und Schwarzenberg, 1905, pp 1002-4.

42. KRAUSE F: Bemerkungen zur operation der hypophysengeschwulste. Dtsch Med Wochenschr 53:691-4, 1927.

43. KRAUSE F: Freilegung der Hipophyse. In Krause F (ed): Die Allgemeine Chirurgie der Gehirnkrankheiten. Part II. Stuttgrat, F Enke, 1914, pp 465-470

44. LANDOLT S: History of pituitary surgery. In Greenblatt SH (ed): A history of neurosurgery. USA, AANS, 1997, pp 373-400.

45. LANZINO G, LAWS Jr ER: Key personalities in the development and popularization of the transsphenoidal approach to pituitary tumors: an historical overview. Neurosurg Clin N Am 14:1-10, 2003.
46. LANZINO G, LAWS ER: Pioneers in the development of transsphenoidal surgery: Theodor Kocher, Oskar Hirsch, and Norman Dott. J Neurosurg 95:1097-103, 2001.

47. LANZINO G, LAWS ER JR, FEIZ ERFAN I: Transsphenoidal approach to lesions of the sella turcica: historical overview. Barrow Q 18:4-8, 2002.

48. LIU JK, DAS K WEISS MH: The History and evolution of transsphenoidal surgery. J Neurosurg 95:1083-96, 2001.

49. MCARTHUR LL: An aseptic surgical access to the pituitary body and its neighborhood. JAMA 58:2009-11, 1912.

50. PAUL FT, CATON R: Notes of case of acromegaly treated by operation. Br Med J 2:1421-3, 1893.

51. PAULESCO NC: Recherches sur la physiologie de I'hypophyse du cervau; I' hypophysectomie et ses effets. J Physiol Pathol Gen 9:441-56, 1907.

52. ROGOWITSCH N: Die Veranderungen der Hypophyse nach Entfernung der Schilddruse. Beitr Pathol Anat Allg Pathol 4:453-70, 1889.

53. SCHLOFFER H: Erfolgreiche Operation eines Hypophysentumors auf nasalem Wege. Wien Klin Wochenschr 20:621-4, 1907.

54. SILBERMARK M: Die intrakranielle exstirpation der hypophyse. Wien Klin Wochenschr 23:467-8, 1910.

55. SVIEN HJ, LITZOW TJ: Removal of certain hypophyseal tumors by the transantral-sphenoid route. J Neurosurg 23:603-11, 1965

56. UTIGER RD, PARKER ML, DAUGHADAY WH: Studies on human growth hormone. I. A radioimmunoassay for human growth hormone. J Clin Invest 41:254-61, 1962.

57. WEISS MH: Transnasal transsphenoidal approach. In Apuzzo MLJ (ed): Surgery of the Third Ventricle. Baltimore, Williams and Wilkins, 1987, pp 476-94.

58. YALOW RS, GLICK SM, ROTH J: Radioimmunoassay of human plasma ACTH. J Clin Metab 24:1219-25, 1964.

59. YANIV E, RAPPAPORT ZH: Endoscopic transseptal transsphenoidal surgery for pituitary tumors. Neurosurgery 40:944-6, 1997.

60. ZERVAS NT: Reflections on the surgery of the pituitary. Clin Neurosurg 27:124-32, 1980.

Original recebido em junho de 2005

Aceito para publicação em agosto de 2005

Endereço para correspondência:

Alexandre de Carvalho Galdino

Rua Conselheiro Agostinho, 55, apto. 307

20770-160 - Rio de Janeiro, RJ

E-mail: alexgaldi@terra.com.br 